\title{
Eficacia, eficiencia y situación de permanencia de la mujer en la escuela de ingeniería en sistemas de la ESPOCH
}

Effectiveness, efficiency and permanence of women in the computer science department in ESPOCH

Patricio Xavier Moreno Vallejo. ${ }^{1}$, Gisel Katerine Bastidas Guacho. ${ }^{2} \&$ Marco Vinicio Ramos Valencia. $^{3}$

Recibido: 11-12-2019 / Revisado: 19-12-2019 / Aceptado: 05-01-2020 / Publicado: 07-02-2020

\begin{abstract}
.
DOI: https://doi.org/10.33262/concienciadigital.v3i1.1.1131
\end{abstract}

The participation of women in education in areas such as science, technology, engineering and mathematics has improved in recent years. However, according to the United Nations Educational, Scientific and Cultural Organization (UNESCO) there are still significant inequities in women's access to higher education at the local, country and region levels, so the research presence of women in the Systems Engineering career with the objective of reviewing the enrollment history that has taken place in the different academic periods from 1993 to 2019 to determine how effective and / or efficient women have been to complete the pensum of the Systems Engineering degree and get your degree. Additionally, a qualitative study has been carried out based on surveys applied to female students of the race on their own, family and school factors. The results indicate that $5 \%$ of female students were efficient in the process of their cohort and of this group of 63 women, $3.17 \%$, graduated as engineers during the 6 months after the end of the curriculum that is optimal. On the other hand, the number of women who have graduated during these 26 and a half years of existence of the career since it was created are $45.61 \%$ and therefore have been effective.

Keywords: Education, women, technology, effectiveness, permanence

\footnotetext{
${ }^{1}$ Escuela Superior Politécnica de Chimborazo, Facultad de Administración de Empresas. Riobamba, Ecuador, pxmoreno@espoch.edu.ec

2 Tecnologías de la Información, Quito, Ecuador, gis.bastidas@outlook.com

3 Escuela Superior Politécnica de Chimborazo, Facultad de Informática y Electrónica. Riobamba, Ecuador, vi_ramos@espoch.edu.ec
} 


\section{Resumen.}

La participación de las mujeres en la educación en áreas como ciencias, tecnología, ingeniería y matemáticas ha mejorado en los últimos años. Sin embargo, según la Organización de las Naciones Unidas para la Educación, la Ciencia y la Cultura (UNESCO) aún existen importantes inequidades en el acceso de mujeres a la educación superior a nivel local, de país y región, por lo que se investiga la presencia de la mujer en la carrera de Ingeniería en Sistemas teniendo como objetivo revisar el historial de matrículas que se han dado en los diferentes períodos académicos desde 1993 hasta 2019 para determinar qué tan eficaces y/o eficientes han sido las mujeres para lograr terminar el pensum de la carrera de Ingeniería en Sistemas y obtener su título. Adicionalmente, se ha realizado un estudio cualitativo en base a encuestas aplicadas a mujeres estudiantes de la carrera sobre factores propios, familiares y escolares. Los resultados indican que el $5 \%$ de mujeres estudiantes fueron eficientes en el proceso de su cohorte y de este grupo de 63 mujeres el 3,17\%, se graduaron de ingenieras durante los 6 meses posteriores al término de la malla curricular que es lo óptimo. Por otra parte, el número de mujeres que se han graduado durante estos 26 años y medio de existencia de la carrera desde que fue creada son $45,61 \%$ y por ende han sido eficaces.

Palabras claves: Educación, mujer, tecnología, eficacia, permanencia

\section{Introducción.}

El presente estudio hace uso de información histórica de la Escuela de Ingeniería en Sistemas (EIS) que es parte de la Escuela Superior Politécnica de Chimborazo (ESPOCH). Las competencias que se pretende conseguir en el estudiante son: el saber aprender relacionado al conocimiento; el saber hacer donde se establecen las aptitudes y destrezas procedimentales; el saber ser a través de comportamientos actitudinales; y el saber convivir con los demás seres humanos. Tiene tres modalidades de graduación que son el examen de grado, proyectos integradores y proyectos técnicos que deben ser realizados como máximo en un año y medio. Se ha realizado rediseños del pensum de la carrera de Ingeniería en Sistemas en los años $1997,2001,2006,2009,2011$ y 2014.

El estudio busca comprender la realidad de la mujer cuando ingresa a estudiar una ingeniería en un ente de educación superior, tomando como caso de estudio las mujeres que han ingresado a la EIS en busca de obtener un título de ingenieras en sistemas informáticos que les permita tener un futuro profesional. La institución encargada de regular la Educación Superior en el país es la Secretaría Nacional de Educación Superior, Ciencia, Tecnología e Innovación (SENESCYT) quien manifiesta que el 26\% del universo de estudiantes abandonan sus carreras en los primeros semestres, y que en el 2012 el índice de deserción llego al 50\%. (Franco Dueñas, 2017)

En el 2010 los jóvenes de entre 19 y 24 años que no estudiaban ni trabajaban en el país eran el 21\% según refiere el Instituto Nacional de Estadística y Censos (INEC), aquí se incluye a la población que dejó la universidad y posteriormente no consiguió trabajo siendo la mayoría 
mujeres (Rodríguez, 2012). (Sinchi \& Gómez, 2018) indican que una de las carreras que más deserción tiene en la Universidad de Cuenca es la de Ingeniería de Sistemas con un 66,45\% y en la Universidad del Azuay también es la de Ingeniería en Sistemas y Telemática con el $39,66 \%$. Según estadísticas publicadas en el 2014 por el INEC, 1 de cada 4 mujeres de 18 a 24 años no cursa una carrera de estudios superiores porque debe dedicarse a tareas de su hogar, lo cual no ha variado desde el año 2006 (Senescyt, 2015).

Pese a que 15 artículos de la Ley Orgánica de Educación Superior (LOES) se refieren a la igualdad de género, aún hay aspectos pendientes de atención que se focalizan en las mujeres jóvenes dado que siguen existiendo problemas de sexismo y discriminación(Senescyt, 2015). En el foro mujeres en la Educación Superior se puntualizó la necesidad de seguir "feminizando" la matrícula, realidad que ya se nota en universidades y escuelas politécnicas del ecuador, pues para el 2018 contaron con aproximadamente 270.000 mujeres frente a 240.000 hombres. Lamentablemente, también se menciona que esta situación no se replica en institutos técnicos y tecnológicos, en donde se registran 77.000 hombres matriculados frente a 75.000 mujeres. En el caso de docentes la situación es más preocupante, pues aún existe una minoría. En el 2018, las universidades y escuelas politécnicas contaron con 29.033 docentes mujeres y 44.536 hombres. Lo mismo ocurre cuando se habla del ejercicio de poder en los institutos técnicos y tecnológicos, donde en el cargo de rectoras hay 19 mujeres frente a 34 hombres. "Las mujeres siguen siendo mayoría en puestos de servicios y no en cargos de toma de decisiones" (Ecuador Universitario, 2019).

Este artículo está organizado de la siguiente manera: en la sección Trabajos relacionados se discuten estudios que tratan sobre la mujer en la educación superior. En la sección Metodología se describe la metodología para la recolección de datos que permiten el análisis de las estudiantes en la EIS. En la sección Resultados se presentan los resultados de la presencia de las mujeres en la EIS obtenidos mediante el análisis de los datos. En la sección Discusión se comparan los resultados obtenidos con otros estudios y se propone investigaciones futuras. En la sección Conclusiones se establecen las conclusiones de la investigación realizada.

\section{Trabajos relacionados}

En el artículo de (Yansen \& Zukerfeld, 2013) se analiza la exclusión de las mujeres del mundo del software, su baja proporción en los procesos productivos de software y en las aulas donde se enseña informática, así como la renuencia de las mujeres a inscribirse, persistir y graduarse en las carreras universitarias de este rubro, debido a factores venidos desde la niñez relacionados a lo que ellas pueden realizar, hasta en los juguetes con los que puede relacionarse. A nivel universitario no se ha podido probar que influya el diseño, los contenidos y dinámica de la carrera en la presencia de la mujer. Se señala también que a finales de la década de 1970 e inicios de la década de 1980 era muy superior en algunas carreras informáticas la presencia de la mujer. Las mujeres piensan que es poco atractivo trabajar en la industria de las Tecnologías de la Información (TI), porque quienes lo hacen son en su mayoría hombres extremadamente intelectuales e introvertidos, sin importar que existe buenos niveles de ingreso y condiciones laborales. A nivel empresarial el porcentaje de las mujeres involucradas en la programación y desarrollo de software llega apenas a un $24 \%$, pero es claro que las representaciones de la programación como una actividad que no es para las mujeres se 
encuentran bien presentes entre quienes deciden contratar personal de un género u otro. Por otra parte, (Sanz, 2008) indica que cuando los campos académicos son incipientes, las mujeres se animan más a ingresar en ellos, pero a medida que se formaliza y matematiza la disciplina, la población masculina empieza a hacer un ingreso mayor en la misma mientras que la población femenina retrocede, sin embargo, el porcentaje de mujeres que acaban la carrera de Informática con respecto al porcentaje de matriculadas muestra que las mujeres obtienen comparativamente mejores resultados académicos que los hombres. Adicionalmente, durante dicho estudio, la mayoría de los profesores entrevistados señalaron que las estudiantes en las aulas son más trabajadoras que los hombres, y suelen obtener (de media) mejores resultados en cuanto a calificaciones.

(Quintero, 2016) realiza un análisis de la creciente exclusión de las mujeres en la Universidad Nacional de Colombia y presenta estadísticas de su presencia en ciertas áreas de conocimiento como la enfermería, artes, ingeniería química, ingeniería bioquímica, medicina veterinaria que oscila entre el 55\% y $45 \%$ de mujeres y la segregación en otras áreas del conocimiento como es el caso de las ingenierías en las cuales el $70 \%$ tiene la presencia de hombres, donde consta la ingeniería de sistemas, ingeniería mecánica, ingeniería eléctrica. En el contexto global esta universidad en el 2013 contaba con 50000 estudiantes de los cuales mujeres constituían el 37\%, la tasa de absorción se ha reducido ante el aumento de la demanda por cupos, y las mujeres han resultado excluidas en mayor proporción que los hombres. En el 2013 se graduaron de la Universidad un total de 7578 personas, donde el 57\% fueron hombres y el $43 \%$ mujeres, si bien la mayor proporción de hombres se mantiene mayoritaria, ésta se reduce en relación con el número de estudiantes matriculados. En un estudio realizado por (García-Holgado et al., 2017), plantean a los estudiantes de la asignatura de Ingeniería de software I una serie de preguntas relacionadas al género, llegando a la conclusión de que sus estudiantes no eran conscientes de la brecha de género más allá del bajo número de mujeres en el curso. (Fernández et al., 2006) confirman el descenso de las mujeres que estudian Ingeniería Informática en la Universidad del País Vasco que en un principio eran cercanas al 30\% en 1998 a tener alrededor del 19\% de matrícula en el 2004, entre las causas que se consideran son factores culturales, baja autoestima que lleva a las mujeres a infravalorar sus capacidades y habilidades.

(Oliveros et al., 2016) manifiestan que, del total de estudiantes matriculados en ingeniería en México, solo el 30\% corresponde a mujeres y del estudio realizado en la Universidad Autónoma de Baja California encuentran que la motivación para seguir una carrera de ingeniería está dada por el apoyo familiar. Adicionalmente, proponen la utilización del modelo Ciencia, Tecnología, Ingeniería y Matemáticas (STEM) para incrementar la inclusión, preferencia y matrícula de mujeres en esta área del conocimiento haciendo referencia a la necesidad de que el Gobierno invierta en programas de motivación enfocados a la ciencia y tecnología desde los niveles previos a la educación superior. STEM combina un número de diferentes tipos de contenidos que acentúan las estrategias educativas con el fin de motivar y evitar la deserción de las mujeres en la ingeniería, tales como: estancias en las empresas para la aplicación práctica de los conocimientos adquiridos en la universidad, implementación de talleres vivenciales, lectura crítica, debates, mesas de discusión, pensamiento crítico, ferias y concursos de ciencias, clubes y redes de investigación, así como también talleres y platicas de mentores exitosos en las áreas STEM. 
En los 36 países de la Organización para la Cooperación y el Desarrollo Económico (OCDE) en el 2019 llega al 30\% la matrícula de las mujeres en ingeniería, mientras que, en educación, humanidades y artes, salud y bienestar esta alrededor del 70\% (Ministerio de Educación y Formación Profesional, 2019). Por otra parte, (Griffith, 2010) indica que, durante la universidad, muchos estudiantes cambian de su especialidad planificada a otra, particularmente cuando esa mayoría planificada estaba en un campo STEM por lo cual la persistencia en una de estas especializaciones es mucho menor para las mujeres y las minorías.

(Zabludovsky, 2007) indica que las mujeres han tenido un aumento notable en la participación en la educación superior y su presencia en las universidades ha llegado incluso a rebasar a la de los hombres como muestran los datos de diversos países de Europa y América del Norte como Noruega (61\%), Polonia, Estados Unidos y Portugal (todos ellos con el 57\%) y Canadá (56\%). Referente a América latina, en Uruguay las mujeres representan el 61\%, en Venezuela el $60 \%$ y en México el 51,5\%.

(Tomassini \& Urquhart, 2011) manifiestan que los sesgos entre mujeres y hombres se observan en las matrículas y egresos en la educación universitaria de grado y posgrado y en las trayectorias académicas, especialmente en el acceso a puestos de jerarquía. Los factores presentes, referidos sobre todo a las calificaciones académicas, son el mejor predictor para evaluar la permanencia de las mujeres y los hombres en las ingenierías encontrándose marcadas diferencias según el sexo. Sin embargo, los factores pasados como calificaciones y promedios en la escuela secundaria, los factores a futuro como aspiraciones y proyecciones personales sobre la carrera y las características demográficas ejercen influencias en las diferencias observadas entre la persistencia o abandono de las ingenierías tanto para hombres como para mujeres. También, manifiestan que la matrícula a partir del 2002 en la carrera de Ingeniería en Computación en la Universidad de la República de Uruguay se ha mantenido por debajo del $20 \%$, además presentan estudios donde se observa que en la Universidad de Costa Rica en el 2007 la matrícula de las mujeres es del 16,7\% y en Estados Unidos el porcentaje de mujeres licenciadas en Ciencias de la Computación en el 2004 es del 25\%.

(Mantilla-Falcón et al., 2017) de su estudio en la Universidad Técnica de Ambato establecen que la primacía del género masculino se mantiene bajo el estereotipo "hombre-ingeniero" observándose que en la carrera de Ingeniería en Sistemas el 75,25\% son hombres y el 24,73\% son mujeres.

Si bien se han encontrado trabajos sobre la mujer en la Universidad, no hay ninguno que realice un estudio profundo en las carreras de Ingeniería en Sistemas o de Software sobre la eficacia y eficiencia de la presencia de la mujer y la influencia del entorno familiar y del medio ambiente en el que tienen que desarrollarse para obtener su título en estas carreras.

\section{Metodologia.}

El estudio se divide en las siguientes fases: Recolección de datos, Análisis de datos cuantitativos, y Análisis de datos cualitativos. Para la fase de recolección se empieza por obtener datos cuantitativos de las bases de datos de la ESPOCH correspondientes a 1162 
estudiantes graduados de la EIS desde el año 1993 hasta el año 2019, los datos incluyen información sobre las fechas de ingreso y grado de los estudiantes. Estos datos se utilizaron para obtener el número de estudiantes graduados por semestre desde la primera promoción que fue en el primer semestre de 1998 hasta el primer semestre del 2019 dando un total de 42 periodos académicos. Posteriormente, se extraen los datos del sistema académico para ver cómo ha ido variando el ingreso a primer semestre de las mujeres comparado con el de hombres con el pasar de los años. Asimismo, se analiza el número de mujeres que han sido eficaces y eficientes durante sus estudios, es decir, las mujeres que han logrado graduarse y las mujeres que lo han hecho en un tiempo óptimo, respectivamente.

Después se procedió a recolectar datos cualitativos durante el período marzo - julio 2019 mediante un cuestionario presentado a mujeres estudiantes de la carrera de Ingeniería en Sistemas y la carrera de Ingeniería de Software, que es el resultado del rediseño a la primera carrera que se menciona. El cuestionario que contenía 14 preguntas cerradas y 6 preguntas abiertas fue aplicado a 66 mujeres. El número de la muestra de mujeres a encuestar se obtuvo mediante la fórmula (1)

Donde:

$\mathrm{n}=$ tamaño de la muestra

$\mathrm{N}=$ tamaño de la población $=80$

$\mathrm{Z}=1,96$

$\mathrm{e}=$ error muestral $=0,05$

$\mathrm{p}=0,5$

$\mathrm{q}=0,5$

\section{Resultados.}

Los resultados de la información recolectada de las bases de datos de la EIS, así como de la encuesta para conocer el entorno familiar y la adaptación a la carrera por parte de las mujeres se presentan a continuación.

1. Análisis de estudiantes matriculadas en primer semestre en los diferentes períodos académicos

La carrera siempre ha tenido un periodo de nivelación de un semestre razón por la cual en un inicio el proceso de recolección de datos empezó en noviembre de 1992 con el semestre de nivelación. Sin embargo, para este estudio se considera los datos recolectados a partir del primer semestre del año 1993, dado que los estudiantes que se consideran como parte formal de la carrera son los de primer semestre excluyendo el semestre de nivelación debido a que este semestre solo busca que los estudiantes posean un mismo nivel de conocimientos antes de ingresar a la carrera de Ingeniería en Sistemas.

En este estudio se presentan los resultados del porcentaje de estudiantes tanto mujeres como hombres que ingresaron a primer semestre de la carrera de Ingeniería en Sistemas, desde el período académico Mayo - Octubre 1993 en el cual se matricularon 7 mujeres y 20 hombres 
siendo la diferencia porcentual de 48,15\% hasta el período académico Marzo - Julio 2019 donde la matrícula de mujeres y hombres es 15 y 77 respectivamente y su diferencia porcentual llega a ser del 67,39\% como se puede observar en la Figura 1, habiéndose incrementado la diferencia porcentual entre el ingreso de hombres y mujeres en estos 26 años y medio en 19.24 $\%$.

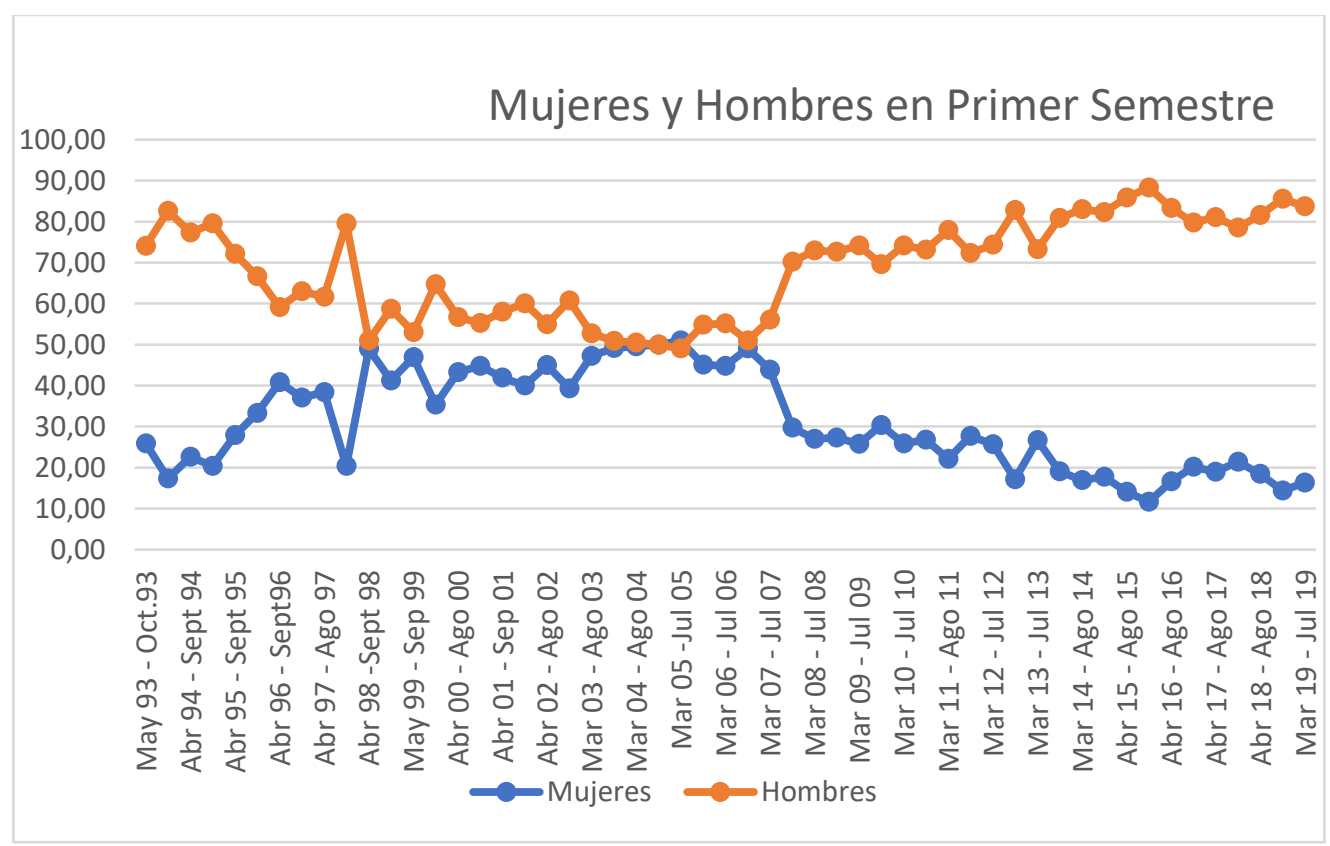

Figura 1: Relación porcentual de estudiantes mujeres y hombres matriculados en primer semestre de la carrera de ingeniería en sistemas

Fuente: Elaboración propia.

Entre los períodos académicos Mayo - Octubre 1993 y Abril - Septiembre 1996 hay un aumento paulatino del número de mujeres en primer semestre llegando a existir una diferencia porcentual del $18.31 \%$, con 29 mujeres y 42 hombres matriculados en el período abril septiembre 1996, luego del cual empieza un disminución hasta que existe una caída abrupta Octubre 1997 - Abril 1998 donde se matriculan 10 mujeres y 39 hombres y la diferencia porcentual llega a ser del $59,18 \%$ que coincide con el período en que se aplica el primer rediseño de la carrera donde se incrementan las asignaturas de 50 a 58, y el período anterior Abril - Agosto 97 es cuando el primer grupo que ingreso llegan a noveno semestre, luego del cual terminan la malla curricular para proceder a realizar la tesis y posteriormente graduarse.

En el período abril - septiembre 1998 es un momento histórico de la carrera en el cual la cantidad de hombres y mujeres tienen apenas una diferencia de $2,04 \%$ debido a que 24 mujeres y 25 hombres se matriculan después de haber aprobado el curso de nivelación. De ahí en adelante la diferencia porcentual se mantiene alrededor del $16 \%$ hasta octubre 2002 - febrero 2003. Posteriormente, existen cinco períodos académicos comprendidos entre Marzo - Agosto 2003 y Marzo- Julio 2005 en los que en promedio la diferencia llega a ser de 1,23\%. En el 2001 
se realiza otro rediseño de carrera donde se implementa el Área Administrativa al incluir asignaturas de esta índole en la malla curricular, además se crea un título intermedio de Analista en Sistemas Informáticos con una formación de seis semestres con el objetivo de hacerle más atractiva a la carrera pues podían los estudiantes obtener doble titulación u obtener un título en menos tiempo. Este rediseño se observa que no le afecta a la carrera y da la impresión de que más bien le hace atractiva para el ingreso de mujeres.

En el período académico septiembre del 2006 - febrero 2007 es la última vez que la matricula entre mujeres y hombres es casi igual teniendo una diferencia de apenas $1,96 \%$ con una matrícula de 50 mujeres y 52 hombres.

Entre junio de 2006 y septiembre de 2007 se realiza un nuevo rediseño de carrera en el cual, además de las asignaturas obligatorias, se incluyen asignaturas optativas y el sistema de créditos. A partir de octubre 2007 - febrero 2008 empieza un decremento de forma considerable de las mujeres existiendo una diferencia porcentual con los hombres de 40,47\% siendo el número de mujeres matriculadas 64 y el número de hombres matriculados 151 .

Se debe tomar en cuenta que en el periodo de gobierno del 2007 al 2017 se realizaron cambios sustanciales en los reglamentos relacionados al ingreso a las Universidades en Ecuador que se encuentran vigentes hasta el día de hoy, en el cual los estudiantes que egresan de los colegios deben dar un examen de admisión y en función del porcentaje obtenido ingresan a cada una de las carreras y universidades del país, pues cada carrera tiene una cantidad de puntos para su admisión.

A partir del período académico marzo - julio 2012 hasta marzo - julio 2019 la cantidad de estudiantes matriculadas ha sido en promedio alrededor de 17 mujeres y 74 hombres, existiendo una diferencia porcentual del $62,46 \%$.

En el período marzo - octubre 2017 se aplica el último rediseño en el cual se hace una reforma total de la carrera cambiando su denominación a Ingeniería de Software donde cada semestre tiene 800 horas, dando un total de la malla curricular de 8000 horas. Durante este proceso se ubican nuevas asignaturas y se desarrollan nuevos programas analíticos para cada asignatura, se incrementa el número de horas del aprendizaje del inglés a 8 horas semanales. Siendo el porcentaje de diferencia en promedio de matrícula entre hombres y mujeres en estos últimos 4 semestre de $64,72 \%$.

En la Figura 2 se puede observar que la EIS ha tenido el mayor número de estudiantes matriculados en el período septiembre 2009 - Febrero 2010 llegando a un total de 280 estudiantes conformados por 85 mujeres y 195 hombres, el período que menos estudiantes ha tenido es Octubre 1993 - Abril 1994 con una matrícula de 4 mujeres y 19 hombres que dan un total de 23 estudiantes.

El promedio de ingreso a primer semestre es alrededor de 31 mujeres y 69 hombres y el promedio global es de 100 estudiantes, en los períodos académicos analizados. Existe un crecimiento sostenido hasta septiembre 2010 - febrero 2011, cuando empieza a descender la 
matrícula hasta septiembre 2012 - febrero 2013, desde el siguiente período académico marzo - julio 2013 empieza a mantener un crecimiento con un promedio de 95 estudiantes, sin embargo, el promedio de mujeres que ingresan se mantiene bajo con un promedio de 17 mujeres comparado al de los hombres que esta alrededor de 78 estudiantes.

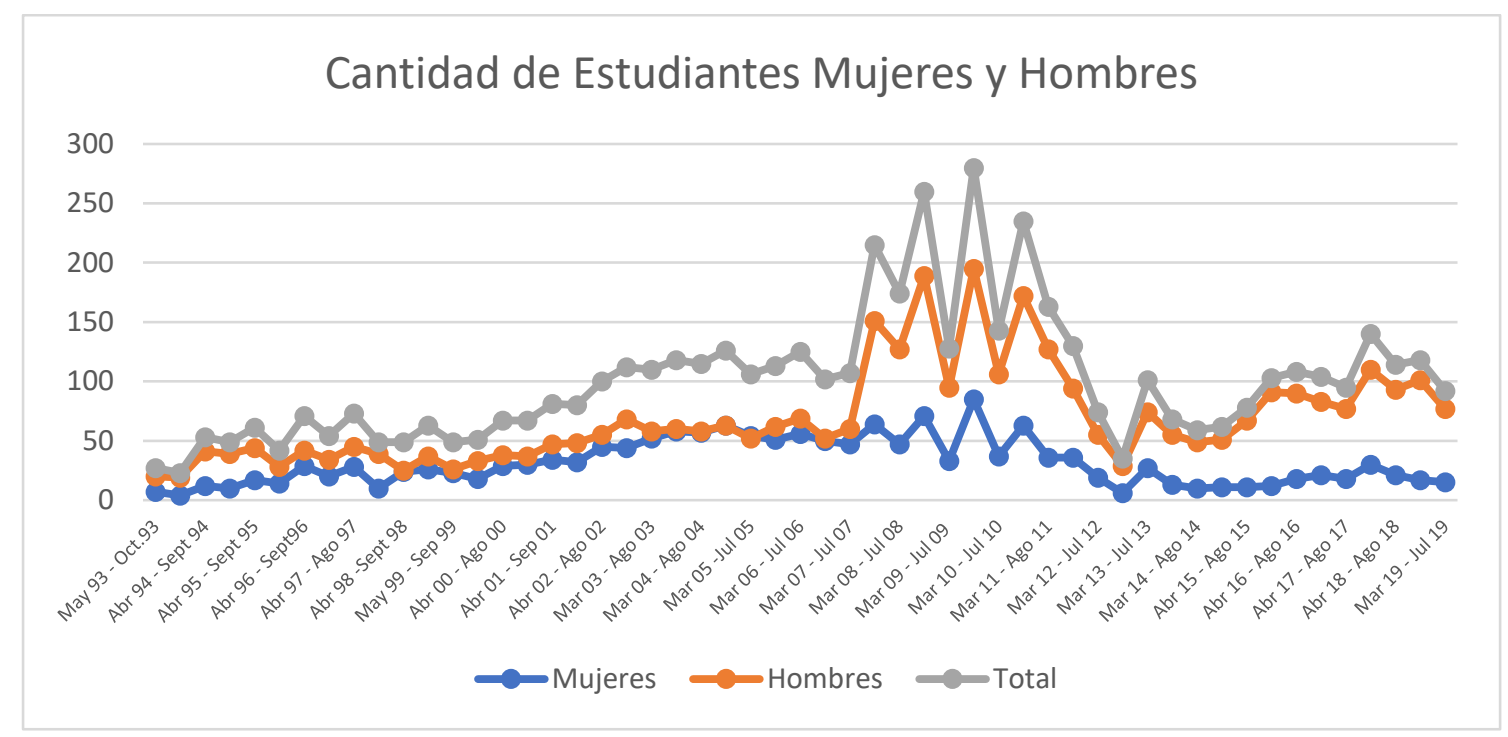

Figura 2: Número de estudiantes mujeres y hombres en cada período académico

Fuente: Elaboración propia.

De igual manera, como se observa en la Figura 3, existe una menor dispersión en el ingreso de mujeres manteniéndose en valores menores al de los hombres, la media de ingresos de mujeres es de 62 mientras que la media de ingresos de hombres es de 135, así también la mediana para las mujeres es de 49 mientras que la mediana para los hombres es de 118.

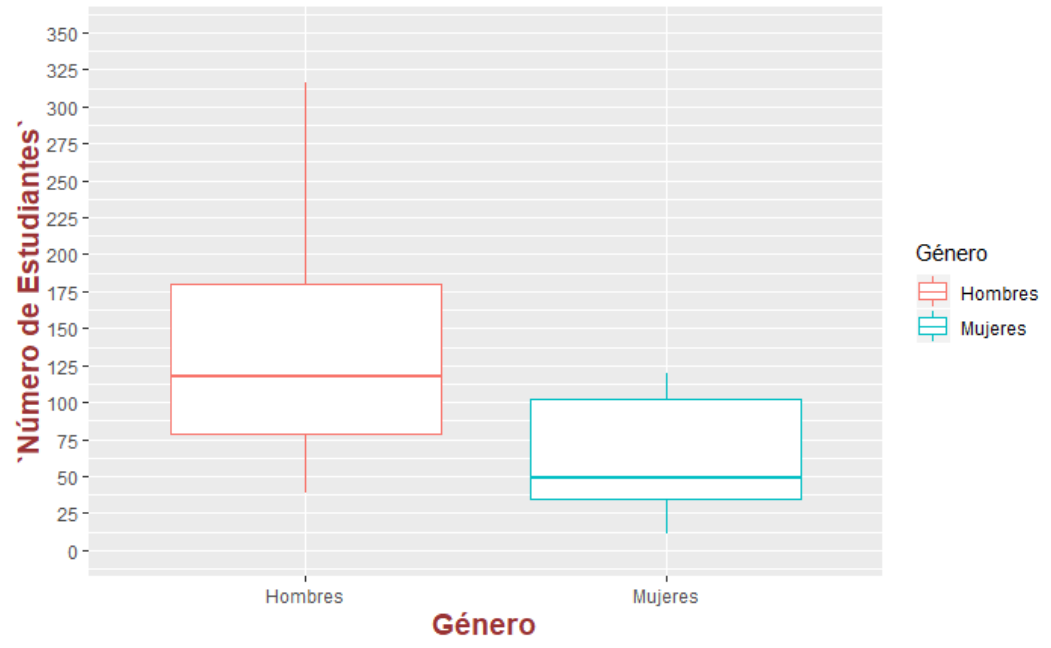

Figura 3: Dispersión en el ingreso de estudiantes a la EIS por género Fuente: Elaboración propia. 
2. Cohortes de periodos comprendidos entre primero y noveno semestre

En la Figura 4 se muestran las cohortes que indican el número de estudiantes mujeres que ingresaron a primer semestre y las que llegaron a noveno semestre sin reprobar ninguna asignatura desde el período mayo - octubre 1993 hasta marzo -julio 2019 dando un total de 45 cohortes.

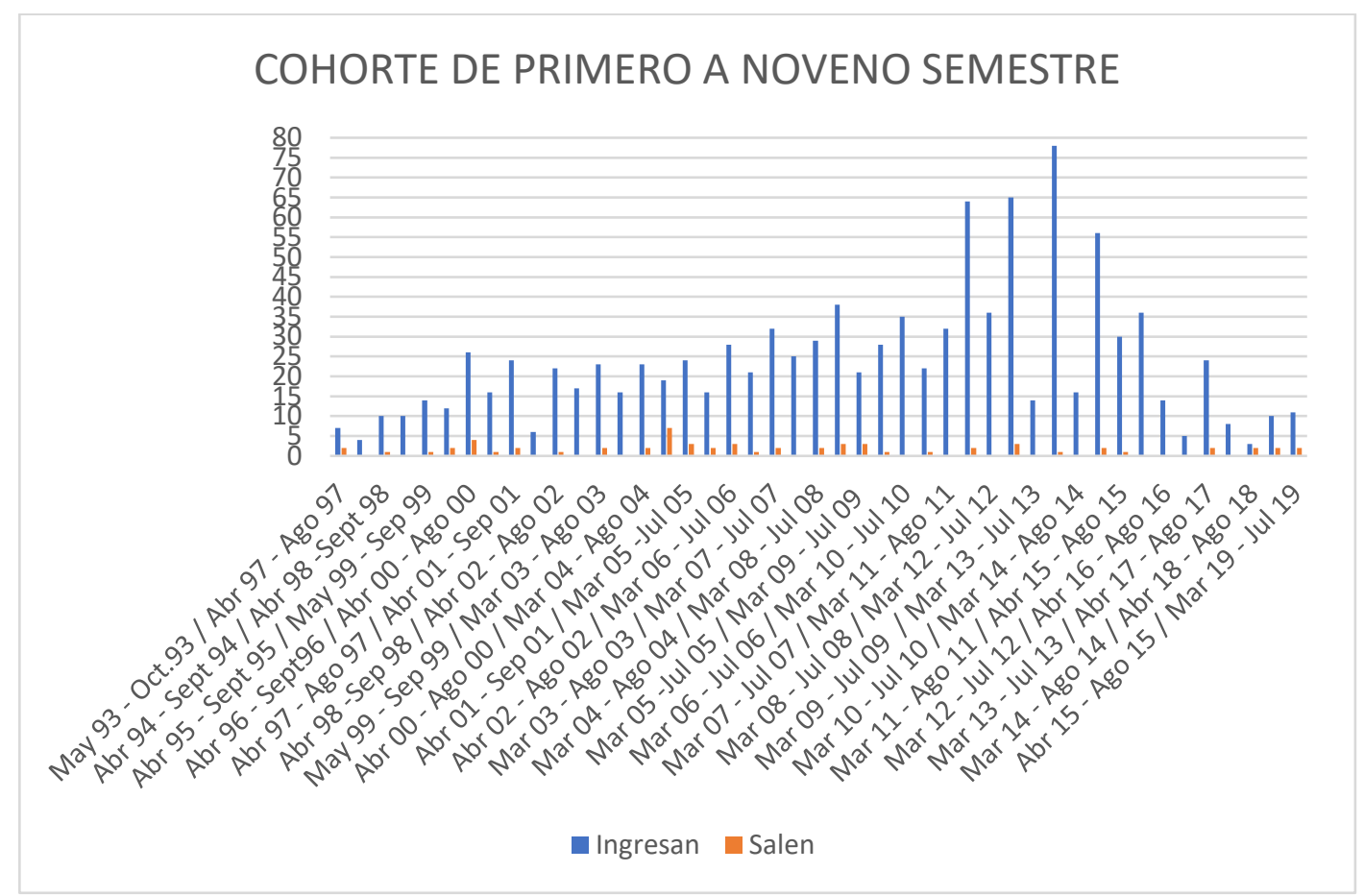

Figura 4: Ingreso de Mujeres a primer semestre y cantidad que lograron llegar a noveno semestre y lo aprobaron sin haber reprobado ninguna asignatura

Fuente: Elaboración propia.

El total de estudiantes mujeres en estas cohortes son 1070, sin embargo, solo 63 estudiantes llegan a noveno semestre sin reprobar ninguna asignatura lo que representa el 5\% del total de mujeres quienes vienen a ser eficientes en el proceso académico dado que cumplieron el objetivo de egresar de la carrera en un tiempo óptimo. La cohorte en la cual ingresaron más mujeres a primer semestre fue la que empieza en el semestre de septiembre 2009 - febrero 2010 con un total de 78 mujeres, la cohorte en la que menos mujeres ingresaron fue la que empieza en el semestre de marzo - agosto 2014 con un total de 3 mujeres, y el promedio de ingreso es de 24 mujeres.

En la cohorte de octubre 2000 - marzo 2001 a septiembre 2004 - febrero 2005 es cuando la mayor cantidad de mujeres de la cohorte llegan a noveno sin reprobar ninguna asignatura en un número de 7 . Por otra parte, el promedio de estudiantes mujeres por cohorte que llegan a noveno semestre sin reprobar es de 1,4 estudiantes, la cantidad es afectada porque existen cohortes en las que no se registra ninguna mujer en noveno semestre bajo la condición analizada. De las 63 mujeres, 2 se graduaron 5 meses después de haber egresado y el tiempo de graduación promedio de este grupo de mujeres es de 21 meses. 
Hasta el período mayo - julio 2019 se han graduado y obtenido su título de Ingenieras en Sistemas Informáticos un total de 488 mujeres que constituye el $45,61 \%$ del total que ingresaron a primer semestre (1070 mujeres). La cantidad de hombres graduados son 673 que representan el 28.64\% de los 2350 hombres que ingresaron a primer semestre. En la Figura 5: Porcentaje de hombres y mujeres graduadas en función de los estudiantes que ingresan, se puede observar que las mujeres son más eficaces que los hombres en el proceso de lograr mantenerse en la carrera y graduarse en función del número de estudiantes que ingresan a la carrera.

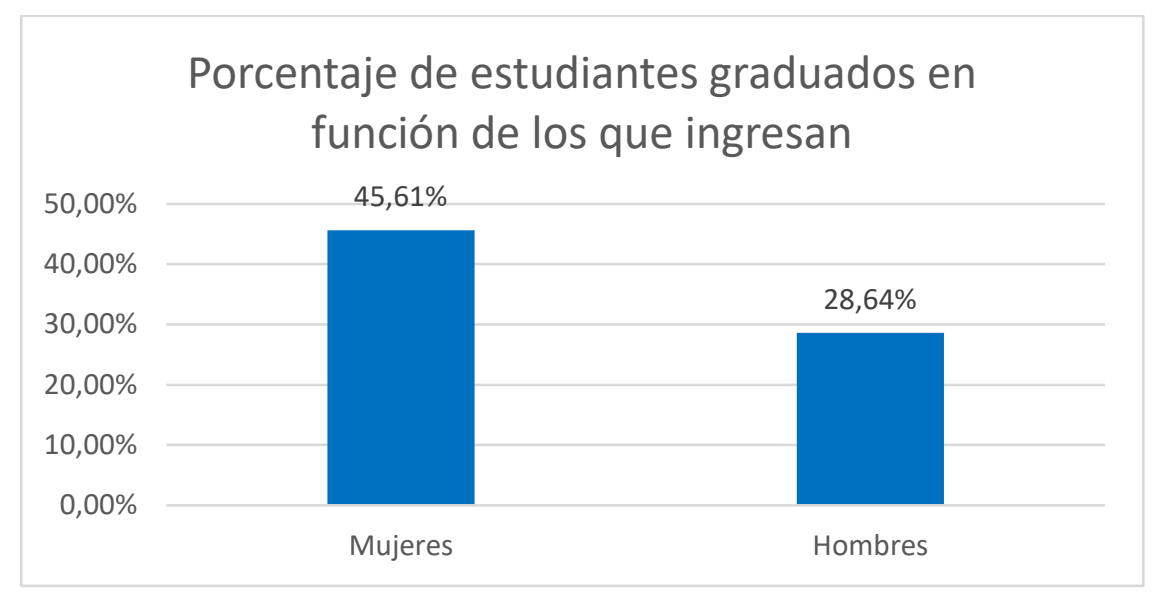

Figura 5: Porcentaje de hombres y mujeres graduadas en función de los estudiantes que ingresan

Fuente: Elaboración propia.

3. Análisis de las encuestas sociodemográficas

Existen factores que pueden o no influir en el ingreso y deserción de las mujeres en una ingeniería, por lo que en base a las encuestas sociodemográfica realizadas a las estudiantes de la EIS durante el período académico marzo-julio 2019 se pretende determinar las razones de la baja de mujeres.

Según la Unesco, el marco ecológico de factores que influyen en la participación, rendimiento y progresión de las mujeres en carreras STEM consta de:

- Factores propios del estudiante como eficacia personal, interés, compromiso, motivación, lenguaje y habilidades espaciales, entre otros.

- Factores Familiares y de pares como bienes y apoyo del hogar, características familiares, creencias y expectativas de los padres, etc.

- Factores Escolares como percepciones de los docentes, interacción docente-estudiante, procedimientos y herramientas de evaluación, etc.

- Factores Sociales como normas sociales y culturales, igualdad de género, legislación y políticas, entre otros.

Por lo tanto, los resultados se muestran en base a los factores propios, familiares y escolares. La Figura 6 muestra los resultados de las preguntas que se relacionan a los factores propios, 
específicamente, se evaluó las razones para escoger la carrera, dificultades antes y durante la carrera, deserción de la carrera y actividades extracurriculares.

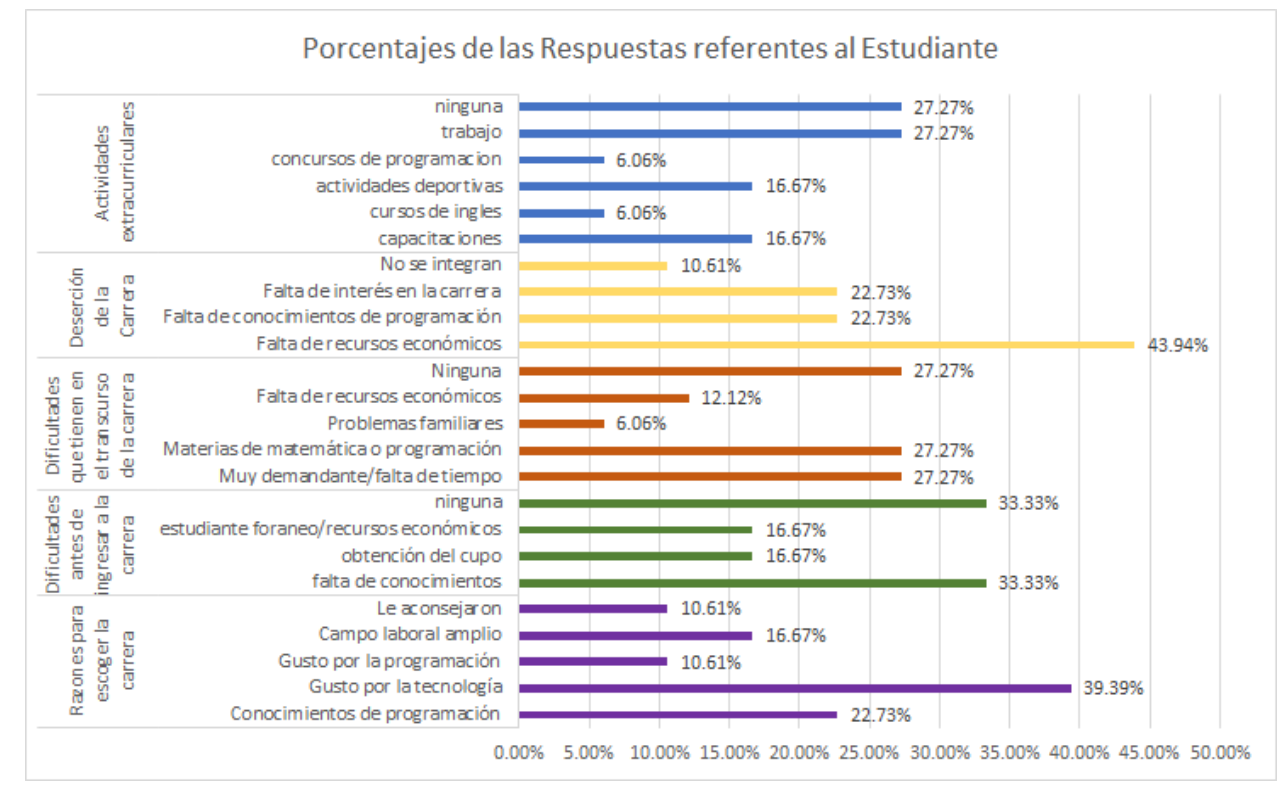

Figura 6. Preguntas referentes a factores propios de las estudiantes

Fuente: Elaboración propia.

Los resultados más relevantes muestran que el 39,39\% menciona que el gusto por la tecnología es la motivación principal para seguir la carrera, así como también un 10,61\% indican que les aconsejaron seguir una carrera relacionada a las tecnologías de la información personas cercanas a su grupo familiar puesto que las mismas siempre estarán vigentes y encontrarán mayores oportunidades de desarrollo personal. Adicionalmente, el 33,33\% de las estudiantes asegura haber tenido problemas por la falta de conocimientos en matemática y física dificultando aprobar el curso de nivelación para el ingreso a primer semestre, el 16,67\% indican que tuvieron dificultad para obtener el cupo pues no alcanzaban el puntaje requerido en el examen propuesto para el ingreso a las universidades y escuelas politécnicas del país, el otro $16,67 \%$ manifiestan que al ser foráneas les ha tocado adaptarse a la ciudad y requieren más recursos económicos para vivir en la misma.

Por otra parte, una vez que han ingresado a la carrera, el 27,27\% de las estudiantes indican que la misma es muy demandante porque existe muchas tareas que son enviadas como trabajos autónomos a ser realizados fuera de horario de clases que les toma un tiempo considerable del día, el otro 27,27\% manifiesta que tiene dificultades en las asignaturas de matemáticas y/o programación debido a que son asignaturas abstractas que requieren razonamiento lógico y usan lenguajes formales. Un $12.12 \%$ de mujeres también menciona que tienen problemas económicos por lo cual no pueden adquirir ciertos materiales necesarios para sus estudios lo cual influye de forma negativa en su desempeño en la carrera. Cuando se les preguntó sobre las causas que están relacionadas con la deserción de mujeres de la carrera, el 43,94\% considera que podría dejar la carrera debido a que provienen de grupos sociales de bajos ingresos económicos que afecta su permanencia en la universidad. El 22,73 \% manifiesta que es debido 
a que no logran superar dificultades relacionadas con la programación; y existe un $22,73 \%$ que ha perdido el interés en la carrera debido a que la misma no ha cumplido con sus expectativas. Por último, existe un $27.27 \%$ de mujeres que no realizan actividades extracurriculares de forma rutinaria, porque la carrera es muy exigente y tienen que dedicarle todo el tiempo que pueden a sus estudios

La Figura 7 muestra los resultados de las preguntas que se relacionan a los factores familiares y de pares.

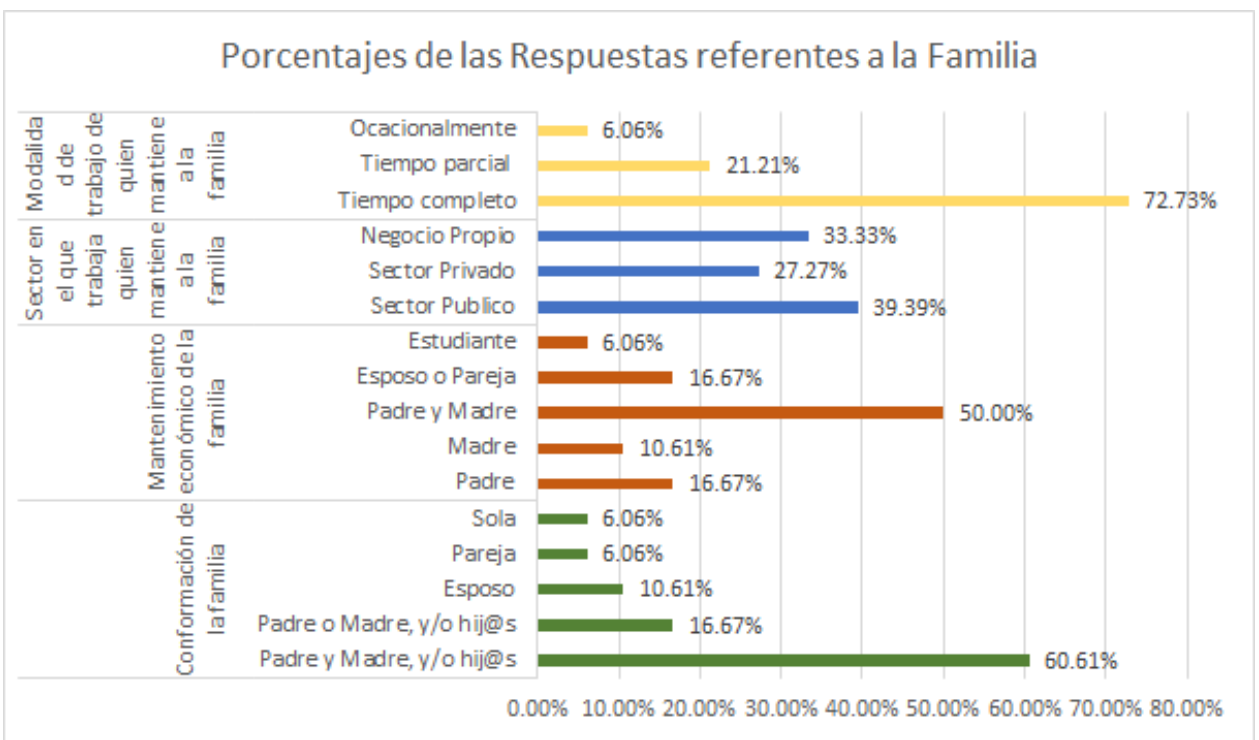

Figura 7. Preguntas referentes a factores familiares

Fuente: Elaboración propia.

El 60,61\% de las mujeres encuestadas pertenecen a una conformación de familia propia de los hogares ecuatorianos donde están presentes madre, padre, e hijos, que de cierta forma brinda estabilidad emocional en la mayoría de los casos para realizar estudios en la carrera. Existe un $16,67 \%$ en el cual no está presente uno de los padres. Alrededor del 10,61\% de las estudiantes durante el proceso de estudios en la carrera se han casado y han conformado una familia. Además, existe un $6,06 \%$ de estudiantes que se han alejado completamente de la familia y viven solas.

Los gastos del hogar del $50 \%$ de las familias a las que pertenecen las estudiantes están a cargo del padre y madre aún en casos en que no conviven con los padres. En un 16,67\% de los casos quien cubre los gastos del hogar es el padre mientras que en el 10,61\% la madre es quién corre con los gastos. Existe un 16,67\% de estudiantes que manifiestan que la manutención está a cargo de su esposo o pareja. Y el 6,06\% de mujeres indican que deben trabajar para poder mantenerse y continuar con sus estudios. Según el informe de la Unesco, tener un nivel socioeconómico estable está asociado a que los estudiantes obtengan mejores calificaciones puesto que puede estar relacionado a que los padres dan un mayor apoyo en el aprendizaje con mayores expectativas académicas.

Por otra parte, la formación académica de los padres y/o familiares es un factor importante, puesto que puede influenciar en la elección de una carrera profesional. En la encuesta realizada 
se analiza el nivel de estudios del padre, madre, pareja y de quién mantiene el hogar como se muestra en la Figura 8. El 63,64 \% de los padres no tienen educación de nivel superior por lo tanto solo el 36,36\% de los padres han obtenido un título profesional; En el caso de las madres, solo $22,73 \%$ han realizado estudios universitarios, por lo que el 77,27\% de las madres no tienen educación de nivel superior. El 78,79\% de las estudiantes mujeres no están casadas, el 21,21\% de las estudiantes están casadas o tienen pareja con la que conviven, bajo ésta última condición el 42,86\% de sus parejas han culminado el bachillerato, y el otro 57,14\% están siguiendo una carrera universitaria o la han terminado. Los resultados obtenidos están en contraste con los resultados de la Unesco, donde se indica que la presencia de familiares con una educación de nivel superior tiene influencia sobre la elección de las mujeres por dichas carreras (UNESCO, 2019).

Ante la consulta en general del nivel de estudios que tiene el familiar que mantiene la familia sin especificar si son el padre y/o la madre, abuelitos, pareja o tíos etc., se observa que el nivel de educación de estas personas esta alrededor de 45,45\% con estudios secundarios, 9,09\% primarios y 9,09\% sin ningún estudio, esto da una idea de la economía que se maneja en los hogares de los cuales vienen la mayoría de las estudiantes de la EIS que al haber hecho estudios máximo de bachillerato conforman familias de nivel económico medio y tendiendo a bajo y el $36,37 \%$ corresponde a familias que al tener educación superior se encuentran en el grupo económico que en el Ecuador se conoce de nivel medio, esto da la idea de la sindéresis que se maneja en el país, si se relaciona la educación con el poder adquisitivo, esto es a mayor educación mayor poder adquisitivo. De estos individuos, el 72,73\% tiene un trabajo a tiempo completo lo que de alguna forma ofrece estabilidad a las estudiantes, pero el 27,27\% de quienes mantienen a las estudiantes tienen trabajos a tiempo parcial $(21,21 \%)$ y ocasional $(6,06 \%)$ complicando la situación de las estudiantes que dependen de estos ingresos económicos y en muchos casos siendo la causa del retiro de la carrera al convertirse en un grupo vulnerable.

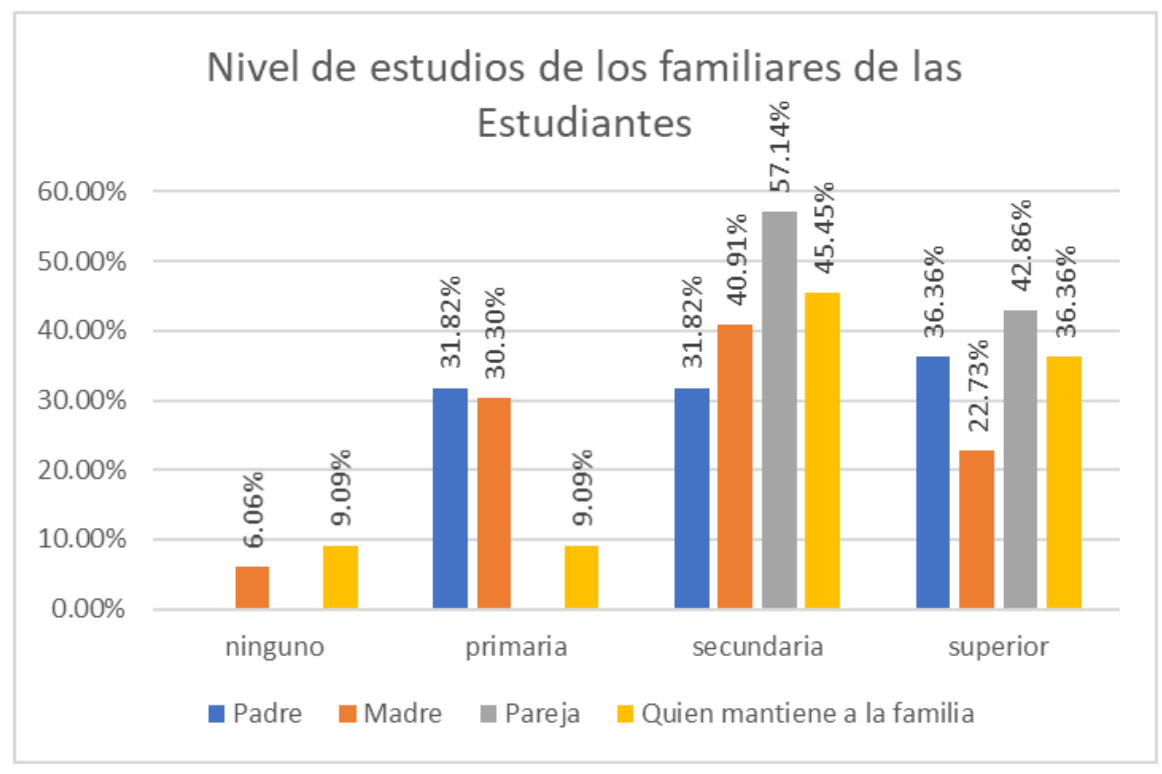

Figura 8. Nivel de estudios de los familiares de las estudiantes Fuente: Elaboración propia. 
La Figura 9 muestra los resultados de las preguntas que se relacionan a los factores escolares. El 72,73\% de las estudiantes manifiestan que no han participado en ninguna actividad de dirigencia estudiantil por lo que se denota la falta de interés o de apoyo para ser parte de esta actividad extracurricular, el 27,78\% dicen que han participado como: presidentas de curso, tesoreras de la asociación de estudiantes, secretarias de la asociación de estudiantes, vicepresidentas de la asociación de estudiantes, o en comisiones de carrera. Por otra parte, en lo que respecta a la capacidad de desarrollo de software de las mujeres, el 10.61\% manifiesta no tener nunca problemas al momento de programar, pues desde que escogieron esta carrera sabían que una de las características de fortaleza de la misma es la programación y por tanto siempre tomaron mucha atención y dedicación a las asignaturas que contemplan el hecho de programar. El $72,73 \%$ indica que casi nunca tienen problemas cuando programan y que constituye una de sus fortalezas la programación porque les parece que es bastante intuitiva y lógica. Un 16,67\% indican que muchas veces tienen problemas en programar esto se debe a que recién están en el proceso de aprendizaje y que esperan en el futuro ir mejorando esta deficiencia. Finalmente, cuando se les pregunto a las mujeres sobre el trato recibido en la institución, el 72,73\% considera que siempre dentro de la carrera han recibe igual trato que sus compañeros hombres por parte de docentes, secretarías y sus mismos compañeros que son con quienes más comunicación tienen. El 27,27\% manifiestan que muchas veces reciben igual trato que sus compañeros hombres pero a veces han sentido que sus compañeros les hacen bullying por el hecho de ser mujer y creer que no van a poder hacer algún trabajo y les toca hacer mayor esfuerzo para demostrar lo contrario. El $72,73 \%$ de las estudiantes consideran que tienen las mismas oportunidades que los hombres en lo que le ofrece la carrera en cuanto a conseguir una beca, participar en concursos, hacer actividades que se presentan en las diferentes asignaturas. El 27,78\% considera que en la mayoría de las veces tienen las mismas oportunidades, pero en algunas pocas ocasiones sienten no tener las mismas oportunidades como cuando han ido a realizar prácticas preprofesionales y no les dan oportunidad de participar o en algún trabajo en clase.

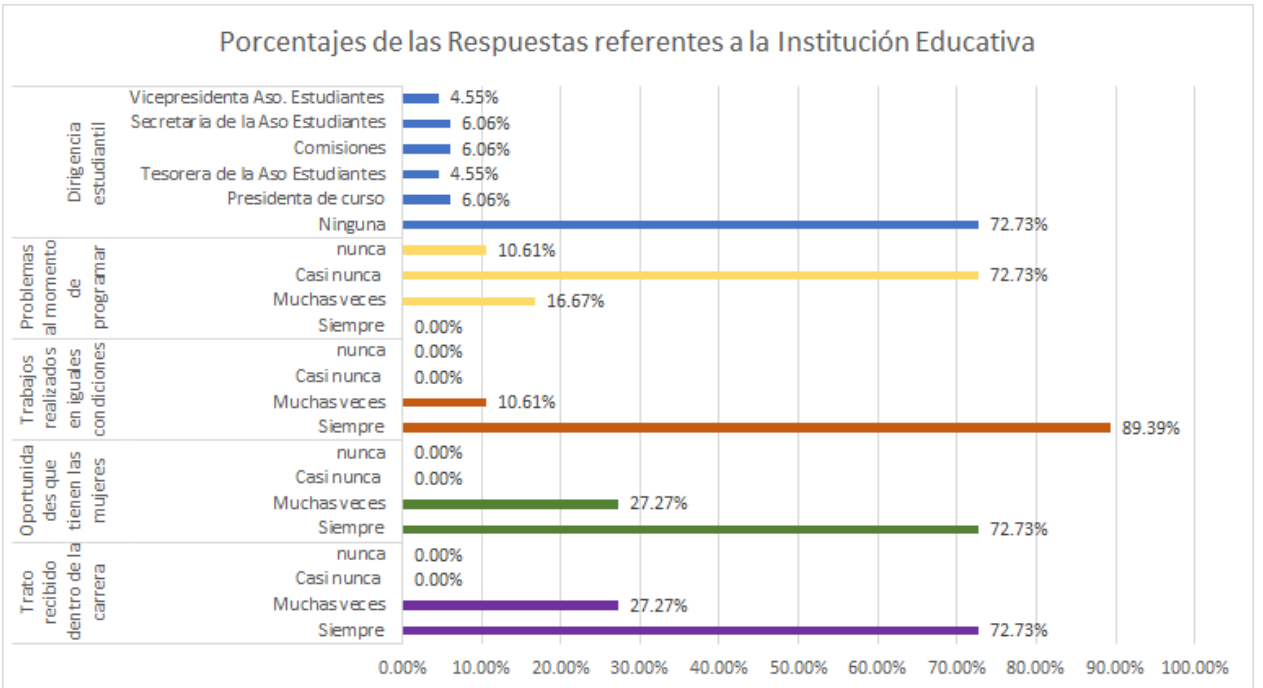

Figura 9. Preguntas referentes a factores escolares

Fuente: Elaboración propia. 
En síntesis, los resultados presentan que existe influencia familiar y del entorno sobre las mujeres para alcanzar el objetivo de finalizar sus estudios superiores y conseguir el título de Ingenieras en Sistemas Informáticos.

\section{Discusión.}

Los resultados obtenidos en el estudio realizado por (Sanz, 2008) indican que a pesar de que hubo un incremento en el ingreso de las mujeres a la carrera de informática en España en los años noventa, no existe un equilibrio entre la cantidad de mujeres y hombres dada la falta de iniciativas que impulsen a mujeres al ingreso de esta carrera. Sin embargo, en dicho estudio no se ha tomado en cuenta los factores que influyen en el desempeño de las mujeres durante sus estudios para determinar su eficacia, eficiencia y permanencia en la carrera. Por lo cual, en este estudio además de realizar un análisis cuantitativo de permanencia de las mujeres en la carrera, se realizó un análisis de los factores propios del estudiante, familiares y académicos para determinar cuáles influyen en el decremento de mujeres en las carreras de TICs. Entre los factores más prevalentes que se pudieron notar están los económicos por lo cual una alternativa para mitigar este problema puede ser la creación de becas exclusivas para mujeres que siguen carreras en el área de las TIC.

Un trabajo futuro a esta investigación puede ser el desempeño y las oportunidades que tienen las mujeres con carreras de TICs en el ámbito laboral contrastando la realidad de las mujeres profesionales que han sido eficaces en el proceso de graduación con las que han sido eficientes. (Hill et al., 2010) indican que el tener un miembro de la familia de género femenino con una carrera de ingeniería influye en la elección de las mujeres por carreras de STEM, sin embargo, en nuestro estudio se evidencia que en la mayoría de casos en el núcleo familiar de las estudiantes no hay profesionales con títulos de tercer nivel. Por lo cual, se ve que la carencia de profesionales dentro de la familia incentiva a las mujeres para alcanzar un título profesional que ayude a mejorar la situación económica de la familia. De igual forma, los resultados obtenidos están en contraste con los resultados de la Unesco, donde se indica que la presencia de familiares con una educación de nivel superior tiene influencia sobre la elección de las mujeres por dichas carreras (UNESCO, 2019).

\section{Conclusiones.}

- En este trabajo se expone un estudio realizado en la EIS desde el año 1993 hasta el 2019 sobre la eficiencia y eficacia que han tenido las mujeres en sus estudios hasta alcanzar el título de Ingenieras en Sistemas Informáticos y se analizan los factores que influyen en el decremento de ingresos.

- De los 54 períodos académicos que han existido en la carrera de Ingeniería en Sistemas solo en 6 períodos académicos, que constituyen el 11,11\% del total, la diferencia entre mujeres y hombres ha sido alrededor del $2 \%$. Por lo tanto, en la mayoría de los períodos siempre la cantidad de hombres ha sido superior que al de mujeres. Solo en el período septiembre 2004 - febrero 2005 las cantidades son iguales siendo de 63, y en el período marzo - julio 2005 es la única vez en que existen más mujeres que hombres siendo 54 y 52 , respectivamente.

- El promedio, el ingreso a primer semestre en estos 26 años y medio ha sido de 31 mujeres y 69 hombres existiendo un $45 \%$ más de hombres que de mujeres, la mayor cantidad de mujeres que han estado en primer semestre sea con primera, segunda o 
tercera matrícula ha sido 85 en el período académico septiembre 2009 - febrero 2010 , y de hombres ha sido 195 coincidiendo el período académico.

- A primer semestre ingresaron 1070 mujeres estudiantes durante los 54 períodos académicos de las cuales 63 estudiantes, que constituyen el 5\% del total, no reprobaron ninguna asignatura en las 45 cohortes consideradas entre primero y noveno semestre. Se han graduado y obtenido el título de Ingenieras en Sistemas Informáticos 488 mujeres que constituyen el $45,61 \%$ del total, de este grupo de mujeres y existe apenas un $0,19 \%$ que lograron obtener su título en los 6 meses posteriores de haber terminado la malla curricular sin haber reprobado ninguna asignatura.

\section{Referencias Bibliográficas.}

Ecuador Universitario. (2019). Mujeres en la educación superior. https://ecuadoruniversitario.com/arte-y-cultura/epn-fue-la-sede-del-panel-mujeres-enla-educacion-superior/

Fernández, V., Ruiz, T., Larraza, E., Maritxalar, M., Lazkano, E., \& Sarasola, K. (2006). Evolución del número de mujeres en la matrícula de los estudios de informática en la Universidad del País Vasco, UPV/EHU. Actas de las XIII Jornadas de Enseñanza Universitaria de Informática, 223-230.

Franco Dueñas, B. de L. (2017). Las causas de la deserción estudiantil durante los primeros dos años en las áreas de Ciencias Sociales y Humanidades en dos universidades de Guayaquil. Repositorio de Tesis - 339. http://cybertesis.unmsm.edu.pe/handle/cybertesis/6188

García-Holgado, A., García-Peñalvo, F., Mena, J. J., \& González, C. (2017). Inclusión de la perspectiva de género en la asignatura de Ingeniería de Software I. http://hdl.handle.net/10366/135405

Griffith, A. L. (2010). Persistence of women and minorities in STEM field majors: Is it the school that matters? Economics of Education Review, 29(6), 911-922. https://doi.org/10.1016/j.econedurev.2010.06.010

Hill, C., Corbett, C., \& St Rose, A. (2010). Why So Few ? Women in Science, Technology, Engineering and Mathematics. En American Association of University Women (Vol. 5, Número 3). http://eric.ed.gov/ERICWebPortal/recordDetail?accno=ED509653

Mantilla-Falcón, L., Galarza-Galarza, J., \& Zamora-Sánchez, R. (2017). La Inserción De La Mujer En La Educación Superior Ecuatoriana : Caso Universidad Técnica De Ambato . 13(2), 12-29. https://doi.org/10.17151/rlee.2017.13.2.2

Ministerio de Educación y Formación Profesional. (2019). Panorama de la educación Indicadores de la OCDE. En Inee. https://doi.org/10.3917/rtm.194.0307

Oliveros, M., Cabrera, E., Valdez, B., \& Schorr, M. (2016). La motivación de las mujeres por las carreras de ingeniería y tecnología. Entreciencias, 4(9), 89-96.

Quintero, O. (2016). La creciente exclusión de las mujeres de la Universidad Nacional de Colombia. Nómadas, 44, 123-145. https://doi.org/10.30578/nomadas.n44a7

Rodríguez, E. (2012). Jóvenes Ecuatorianos en Cifras. http://www.oij.org/file_upload/publicationsItems/document/20121026131329_13.pdf 
Sanz, V. (2008). Mujeres E Ingeniería Informática: El Caso De La Facultad Women and Computer Engineering: the Case of the School of Computer Science At the Technical. ARBOR Ciencia, Pensamiento y Cultura, 184(733), 905-915.

Senescyt. (2015). Participación femenina en la Educación Superior. 66. https://www.educacionsuperior.gob.ec/participacion-femenina-en-la-educacionsuperior/

Sinchi, E. R., \& Gómez, G. P. (2018). Acceso y deserción en las universidades. Alternativas de financiamiento. $\quad$ Alteridad, $13(2), \quad 274-287$. https://doi.org/10.17163/alt.v14n2.2018.10

Tomassini, C., \& Urquhart, M. (2011). Mujeres e Ingeniería en Computación de la UDELAR, Uruguay: Cambios y permanencias. Reporte Técnico RT 11-08, January 2011, 12.

UNESCO. (2019). Descifrar el código. En Descifrar el código: La educación de las niñas y las mujeres en ciencias, tecnología, ingeniería y matemáticas (STEM). UNESCO. https://unesdoc.unesco.org/ark:/48223/pf0000366649?posInSet=1\&queryId=d5f381da $-86 f 6-442 b-8 f 3 b-a 86 a 83220043$

Yansen, G., \& Zukerfeld, M. (2013). Códigos generizados La exclusión de las mujeres del mundo del software, obra en cinco actos. Universitas Humanística, 76(76), 207-233.

Zabludovsky, G. (2007). Las mujeres en México: trabajo, educación superior y esferas de poder. Política y Cultura, 28(1), 9-41. 


\section{Para citar el artículo indexado}

Moreno Vallejo, P. X., Bastidas Guacho, G. K., \& Ramos Valencia, M. V. (2020). Eficacia, eficiencia y situación de permanencia de la mujer en la escuela de ingeniería en sistemas de la ESPOCH. ConcienciaDigital, 3(1.1), 44-62. https://doi.org/10.33262/concienciadigital.v3i1.1.1131

\section{\Ciencia}

El artículo que se publica es de exclusiva responsabilidad de los autores y no necesariamente reflejan el pensamiento de la Revista Conciencia Digital.

El articulo queda en propiedad de la revista y, por tanto, su publicación parcial y/o total en otro medio tiene que ser autorizado por el director de la Revista Conciencia Digital.

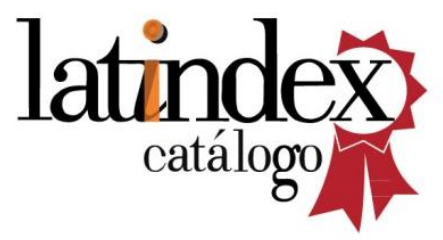

